\title{
Elbow Reconstruction Using Island Flap for Burn Patients
}

\author{
Gi Yeun Hur, Woo Jin Song, Jong Wook Lee, Hoon Bum Lee, Sung Won Jung, Jang Hyu Koh, \\ Dong Kook Seo, Jai Ku Choi, Young Chul Jang \\ Department of Plastic and Reconstructive Surgery, Hallym University College of Medicine, Seoul, Korea
}

Background Deep burns of the elbow lead to soft tissue necrosis and infection, with exposure of deep structures. Adequate wound coverage of this area requires thin, pliable, and durable tissue, while optimal functional recovery requires early coverage and functional rehabilitation. We have found 3 types of island flaps that provide reliable coverage for the elbow.

Methods A retrospective study was performed on all patients who underwent flap coverage of an elbow defect at our hospital. The patients' data including age, sex, cause of injury, wound dimensions, timing of flap coverage, postoperative elbow motion, and complications were investigated.

Results Between 2001 and 2012, 16 patients were treated at our hospital. The mean age was 53.3 years. Three kinds of flaps were performed: 9 latissimus dorsi flaps, 4 lateral arm flaps, and 4 radial forearm flaps. The average defect size was $183.5 \mathrm{~cm}^{2}$ (range, 28 to 670 $\mathrm{cm}^{2}$ ). Wound coverage was performed at mean duration of 45.9 days (range, 14 to 91 days). The mean postoperative active elbow flexion was $98^{\circ}$ (range, $85^{\circ}$ to $115^{\circ}$ ). Partial flap failure occurred in 1 latissimus dorsi flap. Minor complications included partial flap loss (11.8\%), hematoma (23.5\%), seroma (35.3\%), and wound infection (5.9\%).

Conclusions Flap selection for elbow reconstruction is determined by the defect size and the extent of the adjacent tissue injury. Elbow reconstruction using an island flap is a singlestaged, reliable, and relatively simple procedure that permits initiation of early rehabilitation, thereby improving a patient's functional outcome.

Keywords Elbow / Burns / Surgical flaps
Correspondence: Jong Wook Lee Department of Plastic and Reconstructive Surgery, Hallym University College of Medicine, 12 Beodeunaru-ro 7-gil, Yeongdeungpo-gu, Seoul 150-719, Korea

Tel: +82-2-2639-5704

Fax: +82-2-2676-2431

E-mail: jwlpsdoc@yahoo.co.kr
No potential conflict of interest relevant to this article was reported.

\section{INTRODUCTION}

Soft tissue defects around the elbow can be a challenging problem. These are potentially disabling injuries that need early functional rehabilitation, especially following deep burns. Deep burns of the elbow lead to soft tissue necrosis and infection, with exposure of deep structures including nerves, vessels, tendons, and bone. Aggressive debridement should be performed and the defect, which may be extensive, should be covered with well-vascularized tissues. In addition to adequate wound coverage, this area requires thin, pliable, and durable tissue that can endure repetitive motion and external pressure [1]. Optimal functional recovery also requires early coverage and functional rehabilitation.

The choice of coverage depends on a variety of factors including the size and location of the defect, exposure of the vital structures, donor site morbidity, and the general condition of the pa-

Copyright () 2012 The Korean Society of Plastic and Reconstructive Surgeons

This is an Open Access article distributed under the terms of the Creative Commons Attribution Non-Commercial License (http://creativecommons.org/

licenses/by-nc/3.0/) which permits unrestricted non-commercial use, distribution, and reproduction in any medium, provided the original work is properly cited.

www.e-aps.org 
tient. Historically, many treatment options have been proposed for elbow coverage including primary closure, skin graft, local flaps, pedicled flaps, and free flaps; these are usually attempted in a stepladder fashion with the simplest procedures being performed first [2-4]. Multiple options are available for coverage, but the authors have found that three types of island flaps provide particularly reliable coverage for the elbow: the latissimus dorsi, lateral arm, and radial forearm island flaps. Our choice of flap was made based on the extent of concomitant injury to adjacent tissues. In this study, we emphasize the usefulness of island flaps for elbow coverage and provide a treatment protocol for elbow reconstruction.

\section{METHODS}

From January 2001 to February 2012, a retrospective study was performed on all of the patients who underwent flap coverage for an elbow defect except for those with free flaps. Patient data including age, sex, cause of injury, site of injury excluding the elbow, timing of flap coverage, and postoperative elbow motion were examined and wound dimensions were also investigated. In all cases, full wound coverage was not possible with primary closure or skin grafting because of the size and exposure of vital structures. Preoperative computed tomography angiography was performed to check the vascularities of the flaps. Flap coverage was preceded by an aggressive but careful debridement that preserved the vital structures.

Flap selection was based on the defect size and the extent of adjacent tissue injury. Large elbow defects not extending $6 \mathrm{~cm}$ over the olecranon were covered using latissimus dorsi flaps. Small defects of the elbow were covered using lateral arm or radial forearm flaps according to the donor site conditions. The harvesting technique for the flaps followed previously published reports [5]. Each flap was designed so that it was of sufficient size to cover the defect without tension. Postoperatively, the elbow was splinted in a position that avoided stretching of the vascular pedicle. Exercise was started after ten days.

Complications were divided into two groups: major and minor. The major complication was flap failure, which was defined as a partial or total necrosis of the flap that necessitated secondary flap coverage. Minor complications were managed non-operatively and included partial flap loss, hematoma, seroma, and infection. Partial flap loss was defined as partial flap necrosis not requiring additional surgery.

\section{RESULTS}

A total of 16 patients were treated at our hospital between 2001 and 2012; there were 14 males (87.5\%) and 2 females (12.5\%). The mean age of the patient population was 53.3 years (range, 33 to 88 years). The causes of injury were burns in all of the cases: 6 electrical burns (37.5\%), 5 flame burns (31.2\%), 4 contact burns (25\%), and 1 scalding burn (6.3\%). The sites of injury, excluding the elbow, were 8 upper arm injuries (50\%), 4 forearm injuries (25\%), and 2 upper arm and forearm injuries (12.5\%). Two were elbow only injuries (12.5\%). Wound coverage was achieved in a mean duration of 45.9 days (range, 14 to 91 days). The causes of the variation in time to the wound coverage were as follows. The large area burns were treated by skin grafting first $(n=5)$.

Reconstruction of the hand and/or wrist defects preceded elbow coverage, particularly in the electrical burn patients $(n=3)$. Serial debridement was needed to ensure wound viability in some electrical burn patients $(n=3)$. Some patients came to the hospital several days or weeks after injury $(n=3)$. The mean postoperative active elbow flexion was $98^{\circ} \pm 8.6^{\circ}$ (range, $85^{\circ}$ to $115^{\circ}$ ) (Table 1). A total of 17 flaps were performed for coverage in 16 patients. Three kinds of flaps were performed: 9 latissimus dorsi muscle or myocutaneous island flaps (53\%), 4 lateral arm island flaps $(23.5 \%)$, and 4 radial forearm island flaps (23.5\%). The average defect size was $183.5 \pm 195.5 \mathrm{~cm}^{2}$ (range, 28 to $670 \mathrm{~cm}^{2}$ ). The average wound dimension for each flap was $310.1 \pm 195.0$ $\mathrm{cm}^{2}$ (range, 105 to $670 \mathrm{~cm}^{2}$ ) for latissimus dorsi flaps, $31.5 \pm 5.7$ $\mathrm{cm}^{2}$ (range, 28 to $40 \mathrm{~cm}^{2}$ ) for lateral arm flaps, and $44 \pm 11 \mathrm{~cm}^{2}$ (range, 30 to $56 \mathrm{~cm}^{2}$ ) for radial forearm flaps (Table 2). One major complication occurred, which was a partial flap failure secondary to distal necrosis of the flap in one latissimus dorsi flap. The flap failure resulted from distal flap congestion and secondary infection. A secondary radial forearm island flap was performed to cover that elbow defect. Minor complications, which included partial flap loss (11.8\%), hematoma (23.5\%), seroma (35.3\%), and wound infection (5.9\%), were treated with dressings.

\section{DISCUSSION}

Deep burns over the 3rd degree lead to full-thickness skin and soft tissue defects with exposure of deep structures. Early coverage of exposed structures should be performed to initiate earlier rehabilitation, thereby improving the patient's functional outcome. A well-vascularized flap is therefore required for coverage of elbow defects and preservation of deep structures.

Many options are available for coverage of defects around the elbow [2,3]. Local random flaps, including advancement flaps, rotational flaps, Z-plasties, and rhomboid flaps can be used to cover small defects where the adjacent skin is healthy and viable. 
Table 1. Patient data

\begin{tabular}{|c|c|c|c|c|c|c|c|}
\hline Case & Sex/Age & Cause of injury & $\begin{array}{l}\text { Wound size } \\
\left(\mathrm{cm}^{2}\right)\end{array}$ & Previous operation & Flap & $\begin{array}{l}\text { Timing of flap } \\
\text { coverage }\end{array}$ & Elbow ROM $\left(^{\circ}\right)$ \\
\hline 1 & $M / 58$ & Electrical burn & 670 & Debridement & LD & 24 day & 95 (10 to 105$)$ \\
\hline 2 & $M / 51$ & BSC (electrical burn) & 105 & Skin graft & LD & $12 \mathrm{mo}$ & 90 (10 to 100$)$ \\
\hline 3 & $M / 62$ & Flame burn & 30 & Skin graft & RF & 91 day & 90 (5 to 95) \\
\hline 4 & $F / 81$ & Contact burn & 450 & Debridement & LD & 14 day & 115 (5 to 120$)$ \\
\hline 5 & $M / 32$ & Scalding burn & 56 & - & RF & 40 day & 105 (5 to 110$)$ \\
\hline 6 & $M / 60$ & Contact burn & 286 & - & LD & 26 day & 105 (5 to 110$)$ \\
\hline 7 & $\mathrm{M} / 44$ & Electrical burn & 140 & Debridement & LD & 18 day & \\
\hline 8 & $\mathrm{M} / 47$ & BSC (electrical burn) & 120 & Skin graft & LD & $17 \mathrm{mo}$ & 85 (10 to 95$)$ \\
\hline 9 & $\mathrm{M} / 47$ & BSC with ulcer (electrical burn) & 28 & Skin graft, sural nerve graft & LA & $18 \mathrm{mo}$ & 85 (15 to 100) \\
\hline 10 & $M / 60$ & Contact burn & 170 & - & LD & 47 day & 105 (5 to 110$)$ \\
\hline 11 & $\mathrm{~F} / 88$ & Flame burn & 30 & Skin graft & LA & 81 day & 100 (5 to 105$)$ \\
\hline 12 & $M / 38$ & Electrical burn & 40 & Skin graft & LA & 75 day & 105 (0 to 105) \\
\hline 13 & $M / 33$ & Flame burn & 28 & Skin graft & LA & 70 day & 105 (10 to 115$)$ \\
\hline 14 & $\mathrm{M} / 44$ & Flame burn & 450 & Skin graft & LD & 57 day & 90 (5 to 95) \\
\hline 15 & $\mathrm{M} / 46$ & Contact burn & 48 & Debridement & RF & 31 day & $100(0$ to 100$)$ \\
\hline 16 & $M / 64$ & Flame burn & 400 & Debridement & LD & 23 day & 95 (0 to 95) \\
\hline
\end{tabular}

Table 2. Wound dimension of each flap

\begin{tabular}{|lccc|}
\hline Flap & Min $\left(\mathbf{c m}^{2}\right)$ & Max $\left(\mathbf{c m}^{2}\right)$ & Mean $\left(\mathbf{c m}^{2}\right)$ \\
\hline LD & 105 & 670 & 310.1 \\
LA & 28 & 40 & 31.5 \\
RF & 30 & 56 & 44 \\
\hline LD, latissimusdorsi flap; LA, lateral arm flap; RF, radial forearm flap. \\
\hline
\end{tabular}

However, these flaps are limited in their mobility and in their ability to cover large defects because of a poor random blood supply $[4,6]$. Local muscle rotational flaps can also be used to cover small defects of the elbow where deep structures are exposed. Several case reports on local muscle rotational flaps have been described [7-9], but these local flaps are limited to smallsized defects, and local muscles often lie within the zone of injury. Historically, distant pedicled flaps obtained from the chest or abdominal wall were used for elbow coverage [4]. However, these have now been almost completely abandoned because of their multiple disadvantages, which include the need for a second operation, prolonged hospital stay, and joint stiffness secondary to prolonged immobilization [4].

When local flaps or pedicled flaps are not appropriate for soft tissue reconstruction, microsurgical free flaps are an attractive option for coverage of the elbow. A free flap can be performed as a single-stage operation and provides good functional and cosmetic outcomes. However, the free flap requires a longer operation time compared to an island flap and requires a skillful microsurgery technique. In particular, electrical burn patients have potential vascular injury that can lead to vessel sclerosis and thrombosis, which increases the risk of flap failure.
The ultimate choice of flap coverage depends on a number of variables including size of the wound, comorbid conditions, and potential donor-site morbidity. In this study, we highlight the usefulness of island flaps for elbow coverage, which are characterized by reliable pedicles, single-stage procedures, and relatively simple techniques for their elevation.

The latissimus dorsi muscle flap has been a workhorse flap for elbow coverage. It consistently provides coverage of moderate to large soft tissue defects of the elbow [10-12]. This is a reliable flap due to its large arc of rotation and robust pedicle [1]. In our series, large elbow defects were covered using latissimus dorsi muscle island flaps (Fig. 1) or myocutaneous flaps. For elbow defects with upper arm injury, we covered the defects with latissimus dorsi muscle flaps. We created an adequate subcutaneous tunnel, without placing pressure on the pedicle, along the posterior aspect of the upper arm. After the flap was inset into the defect, the muscle was covered with a split-thickness skin graft. In 3 patients with soft tissue defects and burn scar contracture, we covered the elbow defects with latissimus dorsi myocutaneous island flaps. On average, the latissimus has been shown to provide soft tissue coverage at 6.5 to $8 \mathrm{~cm}$ distal to the olecranon [11]. Nevertheless, reliable coverage of the elbow beyond the olecranon requires harvesting of the entire length of the latissimus [10-12]. In our series, all of the defects covered with latissimus were within $6 \mathrm{~cm}$ from the olecranon. Although the latissimus had been confirmed to cover elbow defects $8 \mathrm{~cm}$ over the olecranon [11], we covered the elbow defects using the criteria of $6 \mathrm{~cm}$ from the olecranon for preventing distal necrosis of the flap. We performed latissimus dorsi free tissue transfer in 4 patients because the defects extended more than $6 \mathrm{~cm}$ from the 
Fig. 1. Latissimus dorsi muscle island flap with a skin graft

(A) A 44-year-old man who sustained a 22,900 V electrical burn to the left elbow. Immediate escharectomy was performed, and the defect was covered with an allograft, temporarily. (B) After debridement of the allograft and necrotic tissue, the defect was covered with a latissimus dorsi island muscle flap with a meshed split-thickness skin graft. (C) The elbow at postoperative 10 months. (D) Elbow flexion to $95^{\circ}$ (range, $0^{\circ}$ to $95^{\circ}$ ) at postoperative 10 months.
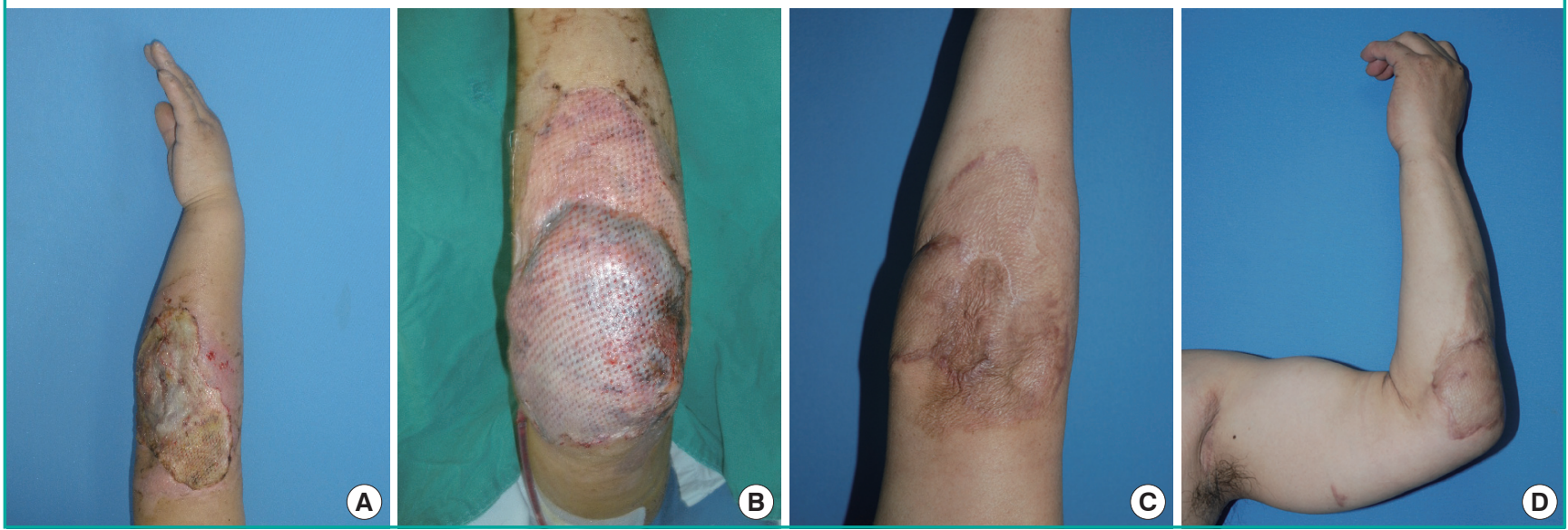

\section{Fig. 2. Radial forearm island flap}

A 62-year-old man had a flame burn to his right arm. (A) The upper arm wound had been covered using a split-thickness skin graft, but he suffered from an unhealed wound on his right elbow. (B) After debridement, a $6 \times 5 \mathrm{~cm}$ sized radial forearm island flap was elevated. The ulnar nerve was dissected (blue background). (C) Functional results at postoperative 14 months. Elbow flexion to $90^{\circ}$ (range, $0^{\circ}$ to $90^{\circ}$ ).
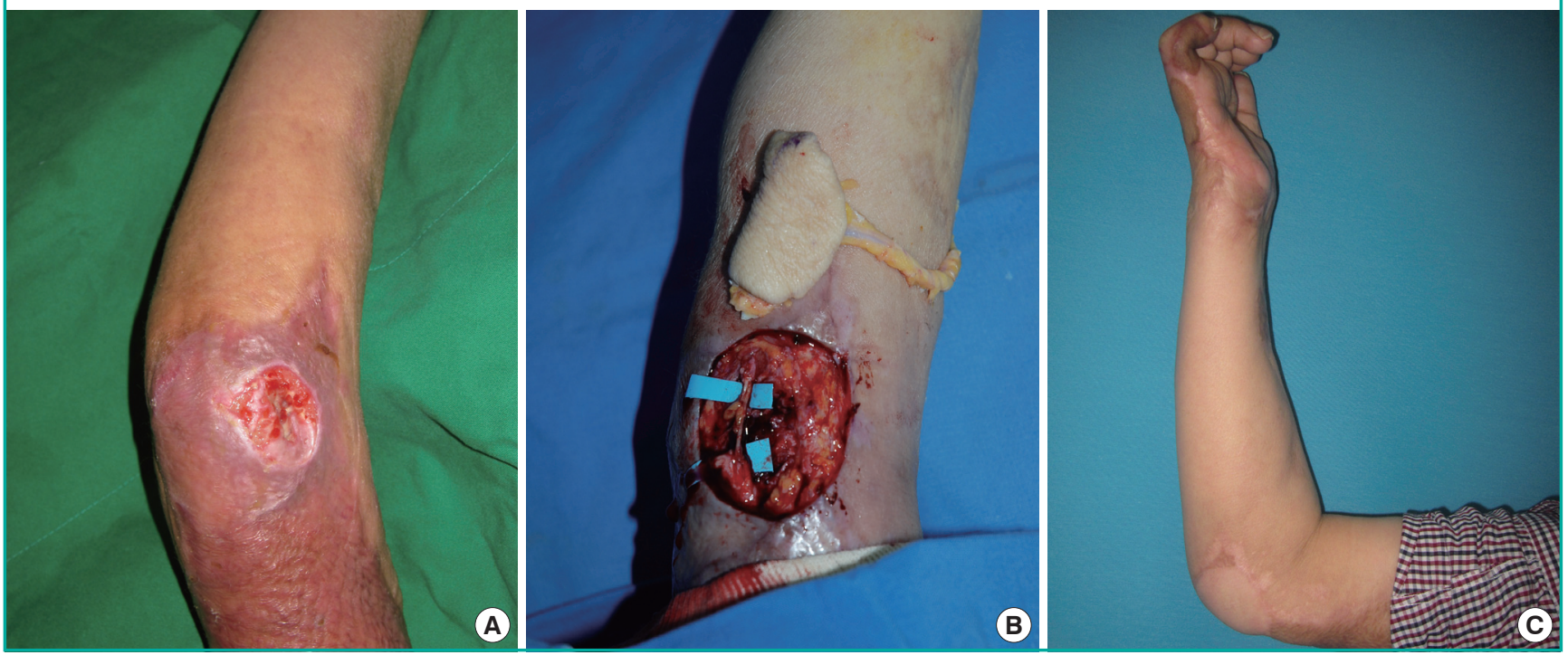

olecranon; these patients were excluded from this study.

Choudry et al. [1] reported that $38 \%$ of the pedicled latissimus dorsi flaps were complicated by necrosis, wound breakdown, or failure. They found that distal flap loss was more common when the flap was extended beyond the olecranon process, which may have been due to the more extensive distal dissection needed to harvest a long flap or because of the excessive tension when positioning the flap in place. Our series found that 1 of $9(11.1 \%)$ of the latissimus dorsi flaps was complicated by flap failure (11.1\%). The failed flap resulted from flap congestion, which might have arisen due to decreased vascularity and tension. Congestion of the distal flap and secondary infection led to flap necrosis, consequently. A secondary radial forearm island flap was performed to cover the defect where the deep structures of the elbow were exposed. However, the remaining eight elbow defects (88.9\%) that extended over the olecranon were covered without secondary operations or wound problems.

The radial forearm flap has been commonly used for coverage of small to moderate size elbow defects. It is a major reconstructive option in elbow coverage because of its flexible arc of rota- 


\section{Fig. 3. Lateral arm island flap}

A 38-year-old man who suffered a 22,900 V electrical burn to his right wrist and elbow. (A, B) After an initial escharectomy, the wrist and elbow defects were covered with an allograft (postburn 2 days). The large volar and dorsal side wrist defect was then covered with an abdominal flap at 26 days after the burn injury. (C) After reconstruction of the wrist defect had been completed, the lateral arm flap was inset into the defect at postburn 2 months. (D) Postoperative view at 12 months; full extension of the elbow.
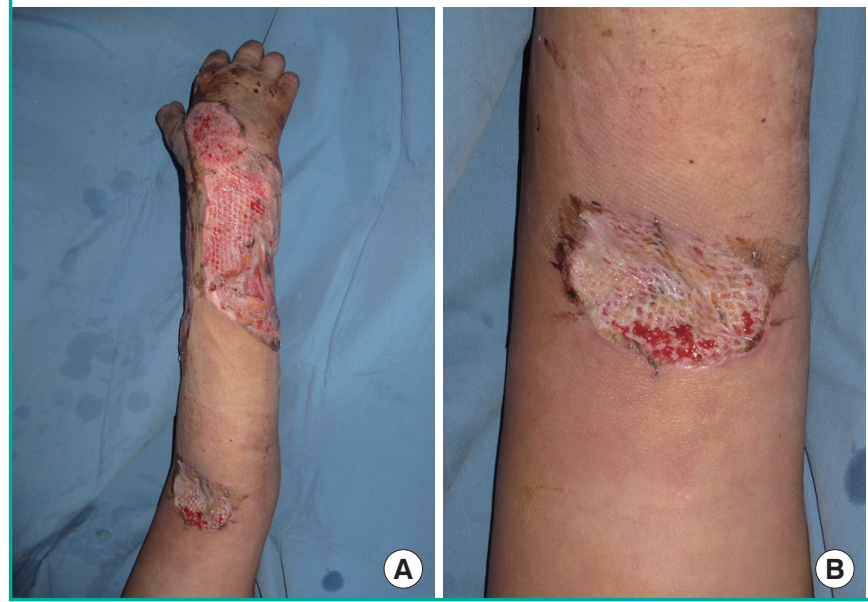

tion, reliable vascularity, versatility, and possible sensory innervations $[2,6,13,14]$. The cutaneous territory covers an area of approximately $15 \times 25 \mathrm{~cm}$ in the volar radial forearm [4]. When the pedicle is mobilized, the flap can be positioned to cover defects on any elbow surface [15]. In our series, 4 radial forearm island flaps were performed without any significant complications (Fig. 2). Minor complications including flap partial loss, infection, seroma, or hematoma were controlled with dressings.

The main disadvantage of the radial forearm island flap is donor site morbidity and sacrificing the radial artery [4]. Donor site morbidity has included cold intolerance, aching, adhesion formation and poor healing, overexposed tendons, and neuroma formation along the superficial radial nerve [14]. However, we experienced only minor complications, such as seroma or hematoma of the donor site, without the other complications listed. Apart from these complications, donor site scarring is always a concern.

The lateral arm flap was used in a small to middle size wound without upper arm injury (Fig. 3). It has several advantages over other flaps, especially its versatility. The lateral arm flap has a reliable anatomy with only minor variations, which do not affect the surgical technique [16-18]. The donor site defect resulting from primary closure is a linear scar on the outer aspect of the upper arm [17]. This seems to be more acceptable than the skin graft covering a forearm donor site [17]. The authors performed a lateral arm flap as a first choice for a small-sized wound without upper arm injury because of less extensive donor site morbidity. The lateral arm flap can cover an elbow defect with an average size of $31.5 \mathrm{~cm}^{2}$ (maximum $40 \mathrm{~cm}^{2}$ ), and it has been reported to have fewer complications when compared to other flaps. The
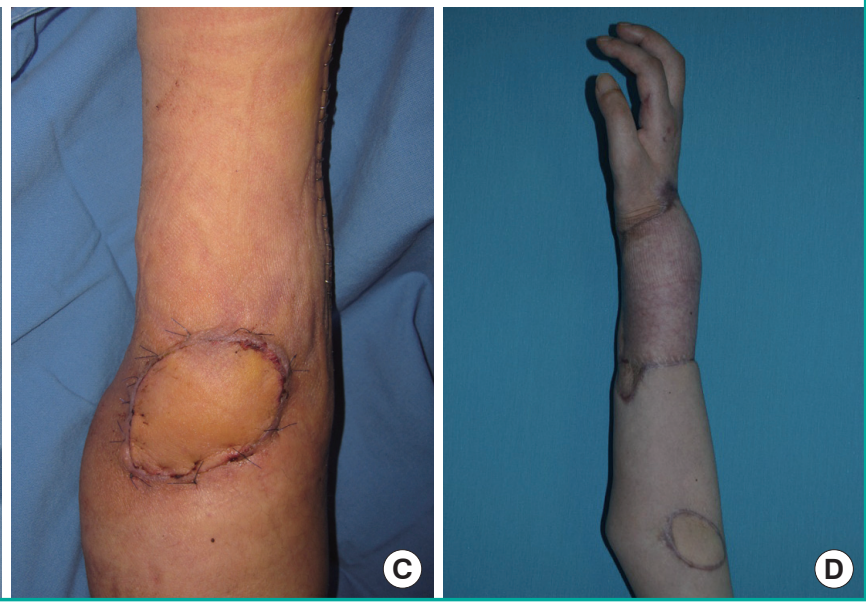

donor site was primarily closed in all of the cases; according to a report, up to an $8 \mathrm{~cm}$ width can be closed [19].

Compared to the radial forearm flap, the lateral arm flap has a size limitation because it can only cover a small wound. The bulkiness of this type of flap, especially from obese patients, can also be a problem when it is used to cover the extensor surface of the elbow. For this reason, in 2 patients with an ulcerated wound over a meshed skin grafted scar, the lateral arm adipofascial flap was elevated as previously described [19]. The adipofascial flap was placed into the defect and then covered with a skin graft. We were able to reduce the bulkiness of flap using this method. If the bulkiness of the flap remains over one year after surgery, it can be reduced by a debulking operation.

The limitation of elbow motion after injury is a well-known complication. A stiff elbow has been defined as an elbow with an extension loss greater than $30^{\circ}$ and flexion of less than $120^{\circ}$ [20]. Morrey [21] found the functional arc of elbow motion during activities of daily living to be $100^{\circ}$ for both flexion-extension $\left(30^{\circ}\right.$ to $\left.130^{\circ}\right)$. The mean postoperative active elbow motion of our patients was $98^{\circ} \pm 8.6^{\circ}$ (range, $85^{\circ}$ to $115^{\circ}$ ). They had no complaints about daily activities, although some patients showed $<100^{\circ}$ of elbow motion $(n=8)$. There are various causes of postburn elbow stiffness such as the type of flap used for coverage, the extent of injury, associated injury, preinjury range of motion, and postoperative rehabilitation [20-22]. All of those factors contribute to the postoperative elbow range of motion. Our series showed that a large defect and late reconstruction time causes the elbow to have less mobility.

Elbow defects in burn patients often occur with concomitant injury to adjacent tissues. The authors classified the coverage 
Table 3. Treatment protocol for elbow reconstruction

\begin{tabular}{|c|c|c|c|}
\hline \multicolumn{2}{|c|}{$\begin{array}{l}\text { Concomitant } \\
\text { injury }\end{array}$} & \multicolumn{2}{|c|}{$\begin{array}{l}\text { Recommended flap according to the size } \\
\left(\mathrm{cm}^{2}\right) \text { of elbow defect }\end{array}$} \\
\hline $\begin{array}{l}\text { Upper } \\
\text { arm }\end{array}$ & $\begin{array}{l}\text { Fore- } \\
\text { arm }\end{array}$ & Small $(<40)$ & $\begin{array}{c}\text { Moderate }(\geq 40,<100) \\
\text { to large }{ }^{a)}(\geq 100)\end{array}$ \\
\hline- & - & LA or RF & RF, LD \\
\hline- & + & LA & LD \\
\hline+ & - & RF & RF, LD \\
\hline+ & + & Free flap & LD, Free flap \\
\hline \multicolumn{4}{|c|}{$\begin{array}{l}\text { +, injured; -, no injury; LA, lateral arm flap; RF, radial forearm flap; LD, latissimus- } \\
\text { dorsi flap. } \\
\text { alff the elbow defect extends more than } 6 \mathrm{~cm} \text { distal to the olecranon, a free flap or } \\
\text { radial forearm flap is recommended. }\end{array}$} \\
\hline
\end{tabular}

method according to concomitant upper arm or forearm injury. The authors' preferred treatment protocol for elbow reconstruction is presented in Table 3.

In conclusion, soft tissue coverage of elbow defects can be a challenging problem for a reconstructive surgeon. Several treatment options are available, but the island flap provides a simple, easy, and reliable solution. For patients with small defects without upper arm injury, the lateral arm flap is recommended. When there is an upper arm injury or scarring, the radial forearm flap can be considered as an alternative method. For patients with larger defects, the latissimus dorsi flap is recommended. When the defect extends more than $6 \mathrm{~cm}$ distal to the olecranon, the radial forearm flap or free flap is recommended. Performing elbow reconstruction using an island flap, which is a single-staged, reliable, and relatively simple procedure, allows initiation of early rehabilitation, thereby improving a patient's functional outcome.

\section{REFERENCES}

1. Choudry UH, Moran SL, Li S, et al. Soft-tissue coverage of the elbow: an outcome analysis and reconstructive algorithm. Plast Reconstr Surg 2007;119:1852-7.

2. Bishop AT. Soft tissue loss about the elbow. Selecting optimal coverage. Hand Clin 1994;10:531-42.

3. Sherman R. Soft-tissue coverage for the elbow. Hand Clin 1997;13:291-302.

4. Jensen M, Moran SL. Soft tissue coverage of the elbow: a reconstructive algorithm. Orthop Clin North Am 2008;39: 251-64.

5. Mathes SJ, Nahai F. Reconstructive surgery: principles, anatomy and technique. New York: Churchill Livingstone; 1997.

6. Stevanovic M, Sharpe F, Itamura JM. Treatment of soft tissue problems about the elbow. Clin Orthop Relat Res 2000;
(370):127-37.

7. Rohrich RJ, Ingram AE Jr. Brachioradialis muscle flap: clinical anatomy and use in soft-tissue reconstruction of the elbow. Ann Plast Surg 1995;35:70-6.

8. Schmidt CC, Kohut GN, Greenberg JA, et al. The anconeus muscle flap: its anatomy and clinical application. J Hand Surg Am 1999;24:359-69.

9. Roukoz S. Musculocutaneous flexor carpi ulnaris flap for reconstruction of posterior cutaneotricipital defects of the elbow. Plast Reconstr Surg 2003;111:330-5.

10. Chang LD, Goldberg NH, Chang B, et al. Elbow defect coverage with a one-staged, tunneled latissimus dorsi transposition flap. Ann Plast Surg 1994;32:496-502.

11. Jutte DL, Rees R, Nanney L, et al. Latissimus dorsi flap: a valuable resource in lower arm reconstruction. South Med J 1987;80:37-40.

12. Sadove RC, Vasconez HC, Arthur KR, et al. Immediate closure of traumatic upper arm and forearm injuries with the latissimus dorsi island myocutaneous pedicle flap. Plast Reconstr Surg 1991;88:115-20.

13. Tizian C, Sanner F, Berger A. The proximally pedicled arteria radialis forearm flap in the treatment of soft tissue defects of the dorsal elbow. Ann Plast Surg 1991;26:40-4.

14. Swanson E, Boyd JB, Manktelow RT. The radial forearm flap: reconstructive applications and donor-site defects in 35 consecutive patients. Plast Reconstr Surg 1990;85:258-66.

15. Timmons MJ. The vascular basis of the radial forearm flap. Plast Reconstr Surg 1986;77:80-92.

16. Lazarou SA, Kaplan IB. The lateral arm flap for elbow coverage. Plast Reconstr Surg 1993;91:1349-54.

17. Katsaros J, Tan E, Zoltie N. The use of the lateral arm flap in upper limb surgery. J Hand Surg Am 1991;16:598-604.

18. Hennerbichler A, Etzer C, Gruber S, et al. Lateral arm flap: analysis of its anatomy and modification using a vascularized fragment of the distal humerus. Clin Anat 2003;16:204-14.

19. Katsaros J, Schusterman M, Beppu M, et al. The lateral upper arm flap: anatomy and clinical applications. Ann Plast Surg 1984;12:489-500.

20. Sojbjerg JO. The stiff elbow. Acta Orthop Scand 1996;67: 626-31.

21. Morrey BF, Askew LJ, Chao EY. A biomechanical study of normal functional elbow motion.J Bone Joint Surg Am 1981; 63:872-7.

22. Morrey BF. The posttraumatic stiff elbow. Clin Orthop Relat Res 2005;(431):26-35. 